\title{
A Heuristic-Based Approach For Usability Evaluation Of ACADEmic Portals
}

\author{
Hmood Al-Dossari \\ Information Systems Department, King Saud University, Riyadh, Saudi Arabia
}

\begin{abstract}
Human computer interaction (HCI) is concerned with the practice of usability, which is a measure that evaluates the simplicity of using a system, software, or interface design for performing particular tasks. Evaluation is a process by which designers gather information about a systems usability to improve its user interfaces features. This research presents a study of a usability evaluation of EduGate, an online academic portal of King Saud University. In this research the heuristic evaluation method was chosen to evaluate the usability of the EduGate. Our results show that, the EduGate suffers from certain usability problem and some recommendations were proposed to enhance the usability of the EduGate. A questionnaire was then used to collect the feedback of real users (i.e. students) on the value of these recommendations. The participants supported $80 \%$ of the proposed recommendations to improve the usability of the EduGate.
\end{abstract}

\section{KEYWORDS}

Usability Evaluation, Usability Problems, Heuristic, Academic Portals.

\section{INTRODUCTION}

A portal is a web-based application that acts as a gateway to information and services from multiple sources that facilitate users' access to the content in one or more repositories [1]. The development of the Internet and World Wide Web have led to many academic institutions promote and encourage optimization of the Internet technology for information dissemination. Higher education institutions (e.g. universities) have developed their own web portals to attract superior students. Manouselis et al. [2] called it educational web portal that serves as a gateway to information and services of some learning or teaching relevance.

EduGate is a Student Portal at King Saud University that provides students with the web based resources they need during their studies. It provides access to University services and information. For example, the EduGate enables students to: view academic calendar, courses registration and check their grades. One main issue need to be considered to provide an easy and effective access to the EduGate is its usability. That is, the extent to which the EduGate can be used by university students to achieve specified goals with effectiveness, efficiency and satisfaction. Figure 1 provides a snapshot of the EduGate (https://edugate.ksu.edu.sa).

Heuristic evaluation [3] is a usability engineering method for finding the usability problems in a user interface design. It involves having a small set of evaluators examine the user interface to judge its compliance with recognized usability principles (the "heuristics"). Evaluation is a process by which designers gather information about a system's usability to improve its user interface's features [4]. In this paper, we examine and evaluate the usability of the EduGate to enable potential suggestions on how its usability can be improved. In particular, the objectives of the study are:

DOI:10.5121/ijcsit.2017.9302 
1. To investigate the methods that can be used to evaluate the usability of the EduGate.

2. To identify major usability flaws in the EduGate.

3. To provide recommendations on how to improve the usability of the EduGate.

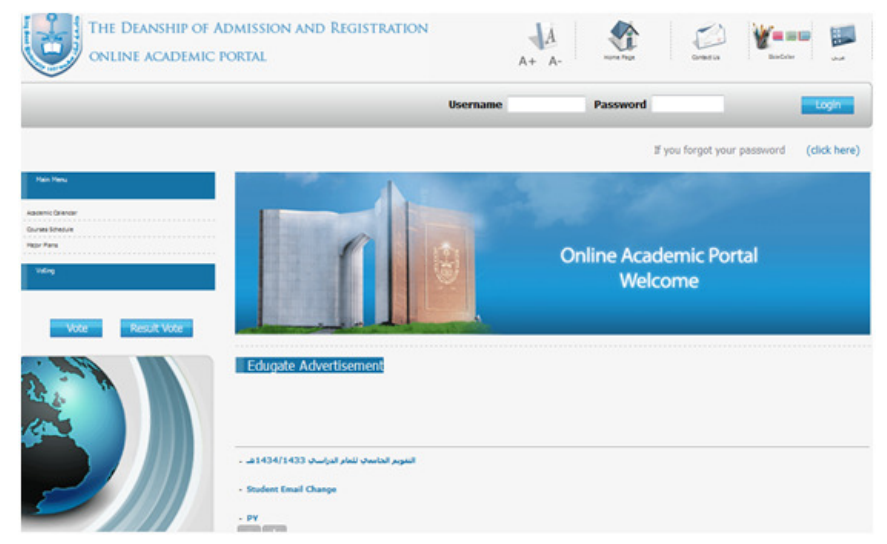

Figure 1: A snapshot of the GUI of the EduGate

The remainder of the paper is organized as follows. Section 2 presents a review of knowledge obtained from previous studies related to the chosen research area. Section 3 investigates the different methods that can be applied to examine and test the usability of an online academic portal applications and the main characteristics of these methods have been highlighted. Section 4 introduces the research methodology and discusses the chosen approach to examine and evaluate the EduGate. Section 5 states the discovered usability problems of the EduGate and provides some recommendations to enhance the usability of the EduGate. Section 6 seeks to evaluate the validity of the recommendations proposed in Section 5. Finally, Section 7 concludes the paper and outlines the future work.

\section{BACKGROUND AND RELATED WORK}

\subsection{Human Computer Interaction}

Human computer interaction (HCI) is a field of study that focuses on improving computer interfaces to make computer systems simple and easy to use. It can be said to be the combining of human user and computer system with the intention of performing a task involving hardware and software components [5]. The HCI occupies a specific point in the intersection between the social and behavioral sciences, as well as between computer and information technology [6]. Improving the interaction between users and computers is the basic goal of HCI. This is achieved by developing appropriate systems that are user-friendly, efficient, and meet the needs of users [7]. Another fundamental goal of HCI is to improve the usability, utility, safety, effectiveness and efficiency of systems that include computers [6].

Similarly, [8] described the HCI goal as ensuring a system is safe and usable, as well as sufficiently functional. Hix and Hartson [5] claim that HCI should provide users with a high level of usability. Developing computers to be user-friendly is one of the biggest challenges in HCI. The development of user interfaces relies upon the cooperation of experts from different disciplines, such as psychologists and computer scientists. While computer scientists attempt to develop high-level programming language, psychologists help by providing information about human capabilities such as human memory and decision making [9]. Interaction design can be 
defined as designing interactive products to support the way people communicate and interact in their everyday and working lives [10]. It is about enhancing the communicative experiences of users in their interaction with computers. In general, people interact with products and computers in four different ways [10]:

1. Direct manipulation, which refers to the physical interaction between people and the computer screen; for example, the user may touch the screen to choose a print action.

2. Language-based, which refers to the communications using verbal words.

3. Demonstration, which displays users' performance in their communications with the real world.

4. Social interaction, where communication takes place implicitly by using body language in the communication.

\subsection{Usability}

Usability is a fundamental factor of human computer interaction. This term was initially used to describe effectiveness by [11], however it was later refined, with two measures: effectiveness and ease of use. While the former measures user performance, the later evaluates interface. Ten usability heuristics were then introduced by Nielsen in 1994 [12]. International standard organization ISO 9241-11 [13] asserts that usability is the extent to which a product can be used by specified users to achieve specified goals with effectiveness, efficiency and satisfaction in a specified context of use. Baecker and Grudin [14] describe usability as a broad concept that basically refers to how easy it is for users to learn a system, how efficiently they can use it once they have learned to use it, and how pleasant it is to use. Nielsen [15] states that the word usability refers to methods for improving ease-of-use during the design process and outlines five quality components to define usability in the following questions:

- Learnability: How easy is it for computer users to perform basic tasks in the first time they encounter the design?

- Efficiency: How quickly can users perform tasks after learning to use the design?

- Memorability: How easily can users re-establish proficiency after a period of absence?

- Errors: How many errors do users make, how severe are these errors, and how easily can they recover from the errors?

- Satisfaction: How satisfied are the users regarding the design?

In summary, usability is a fundamental measure of how users operate the product to achieve their goals and essential in making systems easy to use and easy to learn [16].

\subsection{User Interface}

User interface (UI) is basically an electronic layout with which users directly interact with computer. It consists of two components: input and output. While the former gives the user the ability to enter data, the later produces the computer feedback. Some examples of input devices are a keyboard, mouse and microphone. The output devices include, but are not limited to, screen and printer. It is essential that the UI is designed to be usable by whoever is required to use it in the completion of tasks; they must be able to easily interact with the computer. As described in the previous section, there are a number of ways to determine whether or not a system is good, in terms of product design. If the system is not easy to use, users will refrain from using it. Friedman [17] highlights that designing a good UI is fundamental in meeting users' needs. The design and development of an effective UI can be achieved by gathering information about user's requirements before designing and implementing the UI. Four approaches have been suggested for designing UI [18]: craft, cognitive engineering, enhanced software engineering, and the 
technologist approach. The craft approach considers the design as a piece of art. The cognitive engineering approach uses psychological theory in the design. The enhanced engineering approach encourages UIs designers to understand and consider the main objectives of HCI, whereas the technologist approach uses tools to construct a layout which enables effective interaction between users and application.

\subsection{Previous Studies}

An abundance of literature examines the usability of different applications. For example, Pinelle and Wong [19] studied the usability of video games. They developed heuristics based on problem categories, and then used these to discover problems with usability in video games. An inspection method was adopted using some specialist (evaluators) in the usability inspections process for video games. The evaluators listed several benefits of using heuristics and indicated that it is a good method to test game interfaces. These benefits can assist designers in identifying significant problems which are not always evident with others evaluation methods. Another benefit is that they are relatively in- expensive, easy to use and do not require advanced planning [3]. Dringus and Cohen [20] studied usability attributes in an online learning environment (e.g. navigability, ease of use) to ensure the quality of the learning experience and of the learning environment that serves as an interface for users. They pointed out that instructors can apply user experience in locating specific usability problems in online courses. An adaptable heuristics checklist was used, which included 13 heuristics categories. They stated that not all heuristics can be applied to online courses, but instructors need to carefully select the items applicable to their particular usability evaluation.

Wentz and Lazar [21] discussed the results of a usability evaluation on a desktop and web-based email application used by blind users. Their evaluation involved 15 blind users who tested seven common email applications. Each application was tested between 21 and 35 times and each user evaluated at least two applications. The study concluded that there was scope for improvement. The authors stated that the findings were not limited to blind users, but it should help employers to find an appropriate type of application that fit their needs. They state that their findings would assist employers as they make decisions about the types of email applications that they will use within their organizations.

A systemic review on usability evaluation of game applications for mobile environment was proposed in [22]. The authors investigated around 21 studies on usability of mobile game and highlighted that efficiency, effectiveness, and learnability are the main factors of usability measurement for mobile games. Also, it was found that both expert review and user testing have discovered the most serious playability problems from the user interface.

In [23] the authors designed and implemented a model for a massive online courses (MOOC) on teaching methodologies at Fayoum University, Egypt in cooperation with RWTH Aachen University, Germany. To gauge the usability of the course, they employed evaluation approach based on Conole's 12 dimensions rubrics, ISONORM 9241/110-S as a general usability evaluation, and a custom effectiveness questionnaire reflecting the different MOOC stakeholder perspectives. The results of the study revealed a general satisfaction with the MOOC in terms of usability and effectiveness.

The focus of the study in [24] was the usability evaluation of the Punjab University Library (PUL) website. A comprehensive literature review was conducted to understand the theoretical and technical aspects of the study and data were collected through a questionnaire instrument from 300 respondents and was analyzed by using SPSS. Findings of the study reveal that PUL website proves favourable in two out of five criteria particularly in terms of 'affect' and 
'efficiency'. The data show that affect and efficiency are more outcome oriented than the technical aspects of 'learnability', 'control' and 'helpfulness.'

An online-survey regarding the usability of computer aided manufacturing (CAM) was conducted in [25] and - apart from general usability-effects of age and CAM expertise were analyzed. Main usability barriers were program behaviour and controllability. For older and inexperienced users, cognitive complexity (menu complexity and information density) was found to affect productivity and satisfaction of CAM-software usage. For younger CAM experts, an improved system support (feedback, search function) in solving CAM problems was identified as important requirement. Recommendations for a user-centered CAM software usability optimization were derived.

The purpose of the study [26] was to test smart TV text entry that combines a touch pad and virtual keyboard interaction. A prototype was created and tested against a simple remote control, a touch pad, and a physical keyboard. Twenty college students were recruited to perform a usability test with each of the four different input methods. Participants performed a text entry task and a text edit task on each device. The results indicate that combining a virtual keyboard with touch pad type functionality for text entry and editing can lead to faster text entry and faster text editing.

\section{USABILITY METHODS}

This section describes and reviews the methods of usability measuring commonly employed in the literature, which can broadly be categorized as follows: testing, inspection and inquiry [40]. These methods are summarized in Table 1 and detailed in the following sections.

\subsection{Testing Method}

In testing methods, a selected sample of users works on a real system to assess its usability. Normally, monitoring and video recording are used to observe how the target systems interface supports the users in accomplishing their tasks [28]. In this method, task completion time is captured to allow for users' attitude observation, thus providing the most quantitative data [29]. Testing methods include, but not limited to, the following:

\subsubsection{Thinking-Aloud Protocol}

In this method, participants express their feelings, thoughts and opinions in words during interactions with the system [12]. More specifically, this technique for usability evaluation can be conducted as follows.

1. The participants who will be involved in the usability testing are selected.

2. The tasks and scenarios to be tested in the usability session are determined and prepared.

3. The participants are asked to perform the selected tasks and vocalized their opinions, feelings and thoughts about the system.

This method, however, is not suitable for novice users and too many answers are required from the participants.

\subsubsection{Question-Asking Protocol}

The evaluator asks the participant questions during the evaluation session explicitly in this method rather than implicitly such as in the previous method. That is, the participants are asked to 
freely express their thoughts and feelings without prompting from the evaluator [30]. To do so, the participants are asked to perform specific tasks and then asked questions relating to their experience and interaction with the system to allow the usability evaluator to understand their mental model of the system. More specifically, the question-asking protocol method can be applied as follows:

1. Selecting the participants that will be involved in the test.

2. Deterring the tasks and scenarios that will be used as a test case.

3. Asking the participant to perform a task using the software.

4. During the task, the participants will be asked to expose their thoughts, opinions, and feeling about the target product or software.

However, this method creates a considerable amount of work for both users and evaluators during usability test, i.e. the participants are burdened with tasks to perform. The evaluators are simultaneously pressured by the amount of preparation and questioning required to be asked. Novice users can be frustrated by answering many questions that they may not be well prepared to answer.

\subsubsection{Teaching Method}

In teaching method, usability evaluator asks the participant to teach a new novice participant how to use the system [31]. The evaluator does the following steps to conduct this method: However, neither an expert, the participant who may have no experience about using the target system is in charge to teach the new user (i.e. novice user). This is arguably irrational because the blind (i.e. the participant) is not necessarily able to lead the blind (i.e. the novice user).

1. The participant who will be involved in the test is selected.

2. The target system is determined.

3. The participant is asked to perform a task using the target system.

4. The participant is asked by usability's evaluator to teach a new novice user how to perform the selected task using the system.

5. The interactions between the participant and the novice user are observed and recorded.

\subsection{Inspection}

This technique is widely used in practice since it is both relatively efficient and effective. In this technique, usability experts inspect systems interfaces during formative evaluation [32].

\subsubsection{Heuristic Evaluation}

The heuristic evaluation method is a form of usability engineering conducted to identify the usability problems of user interface design in order to assess its degree of conformance with predetermined usability principles [33, 34]. In this method, several evaluators work independently to come up with potential usability problems by evaluating systems interface. It is essential that the evaluators must work independently, thus not affecting each other. Meanwhile, using several evaluators can be justified by the heuristic evaluation process because it is too difficult for a single person as they would not be able to discover all of the usability problems with an interface [32]. Heuristic evaluation is conducted as follows.

1. Determining usability experts

2. Designing heuristic guide (e.g. usability checklist).

3. Performing an independent evaluation and collecting individual evaluation result.

4. Analysing evaluations and producing usability problems [32]. 
Nielsen and Molich [3] stated that this method is inexpensive and easy to do. However, multiple evaluators are needed because the usability problems are less likely to be detected by a single expert.

\subsubsection{Cognitive Walkthrough}

In this method, one or more usability experts walk through a set of tasks to test the systems usability [35]. It aims to evaluate user interface to support "exploratory learning" i.e., first-time use without formal training. It is strongly recommended to be conducted at early stage of design (i.e. before empirical user testing is possible). The participants perform the walkthrough by asking four questions for each task as following [35] [36]:

1. Will users attempt achieve the required effect? For example, if the task involves printing a document and the first action is to select a printer, then the question is: will they be aware that they need to select a printer first?

2. Will users observe the right button, menu, switch and so on to perform the action? For example, the button on the right for selecting a printer is visible.

3. After users have found the control, will they realize that it creates the effect that they need? For example, the right button may be shown but the user may not understand the icon and so will not click on it.

4. Does the user obtain feedback and understand it? If the right action is performed, will the user understand that they have performed the correct action?

Answering the previous four questions for each task will make the users recognize and discover usability problems. However, when applying walkthrough method caution should be taken. There are two common misunderstanding in conducting walkthrough method [35, 36]:

- Evaluators have no knowledge about how to perform the task.

- The walkthrough does not test real users on the system. This leads to produce fake potential usability problems.

\subsubsection{Pluralistic Walkthrough}

In this method, a group of users, system developers and human factors engineers walk together through a set of primary tasks to discover usability problems [37]. This is so that each member of the group brings a certain perspective, expertise and a set of goals for the system design so enables a greater number of usability problems to detected and maintained. Generally, more people working to test the usability of a system will create a higher probability of any problems being discovered. The efficient interaction between the team is essential to help discover and resolve usability issues faster [12]. This method takes the following steps:

1. One person is nominated to act as group's coordinator.

2. Targeted tasks, paper prototypes, screens shots etc. are presented to the group.

3. Each participant writes down comments on each user interface.

4. After demo, a discussion controlled by the coordinator will be followed to discover and resolve usability issues. 
Table: Summary of reviewed usability methods

\begin{tabular}{|l|c|c|c|c|c|}
\hline \multicolumn{1}{|c|}{ Usability Method } & Method Type & $\begin{array}{c}\text { Number of } \\
\text { evaluators }\end{array}$ & Evaluator(s) & Technique & Comments \\
\hline $\begin{array}{l}\text { Thinking-aloud } \\
\text { Protocol }\end{array}$ & Testing & $>1$ & Participants & Laboratory & Not suitable for novice users \\
\hline $\begin{array}{l}\text { Question-Asking } \\
\text { Protocol }\end{array}$ & Testing & 1 or more & Participants & Laboratory & $\begin{array}{c}\text { Overloads users and evaluators } \\
\text { by a lot of work }\end{array}$ \\
\hline Teachingmethod & Testing & 2 & $\begin{array}{c}\text { Participant }+ \\
\text { novice user }\end{array}$ & Laboratory & Irrational as blind leads blind \\
\hline Heuristic Evaluation & Inspection & $3-5$ & Experts & Exploratory & $\begin{array}{c}\text { Inexpensive, intuitive and easy } \\
\text { to do }\end{array}$ \\
\hline $\begin{array}{l}\text { Cognitive } \\
\text { walkthroughs }\end{array}$ & Inspection & 1 or more & Experts & Exploratory & $\begin{array}{c}\text { Does not test real users on the } \\
\text { system }\end{array}$ \\
\hline $\begin{array}{l}\text { pluralistic } \\
\text { Walkthroughs }\end{array}$ & Inspection & $>=3$ & $\begin{array}{c}\text { Users }+ \\
\text { Engineers }\end{array}$ & Exploratory & $\begin{array}{c}\text { Strong focus on realusers, but a } \\
\text { fairly large people is required }\end{array}$ \\
\hline Field Observations & Inquiry & $>1$ & Experts & Contextual & $\begin{array}{c}\text { Hard to apply and time } \\
\text { consuming }\end{array}$ \\
\hline Focus Groups & Inquiry & $4-12$ & Users & Ethnography & Time consuming and high cost \\
\hline Interviews & Inquiry & $>1$ & Users & Ethnography & $\begin{array}{c}\text { Less time consuming and easy } \\
\text { to apply }\end{array}$ \\
\hline
\end{tabular}

This method is attractive for two main reasons: firstly, there is a strong focus on real users in the task analysis, leading to more problems identified at an early stage of system development. Secondly, the discussion of identified problems in a multidisciplinary team will develop usable and efficient solutions. However, one difficultly in the use of this method results from the vast number and variety of participants required simultaneously, such as users, developers and engineers.

\subsection{Inquiry}

In inquiry methods, instead of observing users working on some predefined tasks by the evaluator such as in testing methods, the evaluator monitors users during their work in the real work context [34]. The main focus of inquiry methods is to collect information about the likes, dislikes and needs of the user in order to understand and consider their preferences in system design. One reason to make inquiry methods attractive and useful is that they allow the evaluator to assess the user, the tasks, and the working environment at the same time [38]. The common usability inquiry methods are described below.

\subsubsection{Field Observations}

Here usability experts observe current and potential users in the workplace during normal using of the system [12]. The usability experts attempt to answer two main questions:

1. What are users mental model? (e.g., their likes, dislikes and needs).

2. Are the users using the system in the expected way?

The general assumption of field observation is that usability evaluation is context-dependent and it should be conducted to capture natural habitat of users to generate appropriate findings.

\subsubsection{Focus Groups}

The main idea of this method is to share the experience between group members by interacting and discussing ideas [30]. This is conducted by producing a variety of ideas about the best way to enhance user interfaces. In contrast to the field observation method, in which users' preferences are captured implicitly, the focus group method can be used to explicitly collect these preferences during the discussion between the group members. While the field observation is primarily 
observation of use, the focus group asks questions about use (i.e. it is ethnographic). However, a focus group is time consuming and relies on the honesty of group members. All aforementioned issues as well as selecting appropriate moderator can lead to higher costs making this method a costly endeavour [39].

\subsubsection{Interviews}

An interview method is similar to focus group, except in that instead of interviewing with a group of users, the interview is normally taken place with one user at a time [29]. This method is efficient when time constraints cannot be accommodated or when observing user behavior cannot be carried out, particularly as it rarely occurs or is unpredictable [40]. Interviews are executed as follows: the interviewer presents a question to the user, the user gives a reply, and the interviewer records user's answers by writing notes or using a recording device and later transcribing it. However, in a similar way to a focus group, it relies on the trust of the participants.

\section{RESEARCH METHODOLOGY}

Figure 2 outlines the main stages of our research methodology. The following sections also describe each stage in details.

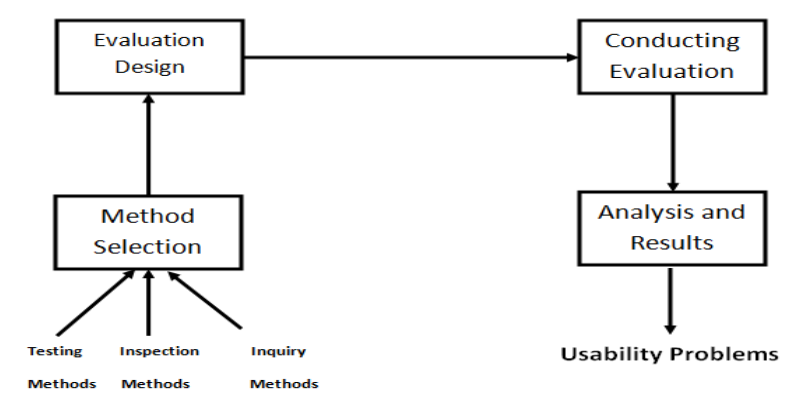

Figure 2: Stages of the Proposed Approach

\subsection{Method Selection}

In our research, heuristic evaluation method adopted for the following reasons:

- Heuristic evaluation is less expensive than other types of usability methods as it requires fewer resources (e.g., from 3 to 5 experts) [33]. As this study is limited in terms of resources, it is reasonable to select and use this method for usability evaluation of online academic portal applications in this work.

- Heuristic does not require formal usability training for evaluators in their study [31, 33].

- Heuristic evaluation provides quick feedback about usability problems making the method the natural choice for this research, due to limitations in time.

- Usability problems discovered by heuristic evaluation method are normally restricted to user interface such as use of colours and consistency of terminology which makes this method is suitable in evaluating online academic portal applications.

- It is generally agreed that there is a degree of overlap between problems identified by heuristic evaluation method and other usability [20]. 


\subsection{Evaluation Design}

Inspired by ten principles proposed by Nielsen [33], the heuristic checklist was designed to test the usability of online academic portal applications. In the following, we describe the 10 heuristic categories and corresponding questions that need to be answered by experts. Each heuristic category has a set of criteria that will be used by evaluators (i.e. experts) in evaluating the EduGate.

1. Visibility of system status. Visibility of system status refers to informing the users what is going on in the system through appropriate feedback within reasonable time [15]. For the visibility status of EduGate, we defined the following criteria:

- Is the selected icon labelled clearly and distinctive from the unselected icons? (Criteria 1.1)

- Does head title clearly describe the page that is open? (Criteria 1.2)

- When the user moves the cursor over a box, is any visual feedback provided? (Criteria 1.3)

- Is any feedback provided when the user presses the function keys (e.g. F1, F2, ... F12)? (Criteria 1.4)

2. Match between system and the real world. The system should speak the users' language, with words, phrases and concepts familiar to the user, rather than system-oriented terms. Follow real-world conventions, making information appear in a natural and logical order [15]. To judge to what extent the EduGate match the real world of students, we defined the following criteria:

- When prompts imply a necessary action, are the words in the message consistent with that action? (Criteria 2.1)

- Is each page of EduGate described clearly for the students? (Criteria 2.2)

- Is the language used in the pop-up windows that display confirmation or error messages clear and simple? (Criteria 2.3)

- Are the meanings of the boxes and icons understandable for both advanced and novice users? (Criteria 2.4)

3. User control and freedom. Users often choose system functions by mistake and will need a clearly marked "emergency exit" to leave the unwanted state without having to go through an extended dialogue. Support undo and redo. We defined the following criteria to examine if the EduGate support student control and freedom:

- Can progressed operations be cancelled by the students? (Criteria 3.1)

- When prompts imply a necessary action, are the words in the message consistent with that action? (Criteria 3.2)

- Is the language used in the pop-up windows that display confirmation or error messages clear and simple? (Criteria 3.3)

- Are the meanings of the boxes and icons understandable for both advanced and novice users? (Criteria 3.4)

4. Consistency and standards. Users should not have to wonder whether different words, situations, or actions mean the same thing. Follow platform conventions. To assess the consistency and standards of EduGate, we defined the following criteria: 
- Are all icons correctly labelled and have the same length? (Criteria 4.1)

- Does each EduGate window have a different title? (Criteria 4.2)

- Is the page title justified? (Criteria 4.3)

- Does the "sign out, sign in" always appear in the top right of the page? (Criteria 4.4)

5. Error prevention. Even better than good error messages are a careful design which prevents a problem from occurring in the first place.

- Is an alert sound used to inform users when an error occurs? (Criteria 5.1)

- Is the user informed of the severity of the error in the error message? (Criteria 5.2)

- Are any suggestions provided about possible reasons for the problem reason in the error message? (Criteria 5.3)

- Do error messages suggest that users correct the error? (Criteria 5.4)

6. Recognition rather than recall. Make objects, actions, and options visible. The user should not have to remember information from one part of the dialogue to another. Instructions for use of the system should be visible or easily retrievable whenever appropriate.

- Is the EduGate prevent the students from making errors? (Criteria 6.1)

- Is an alert sound used to inform students when an error occurs? (Criteria 6.2)

- Are icons separated by at least one space? (Criteria 6.3)

7. Flexibility and efficiency of use. Accelerators - unseen by the novice user - may often speed up the interaction for the expert user such that the system can cater to both inexperienced and experienced users. Allow users to tailor frequent actions.

- Are there any options in each page for students to course add, dropped or exist? (Criteria 7.1)

- Is the most important action or icon used as the first in each menu? (Criteria 7.2)

- Is the size of each icon suitable? (Criteria 7.3)

- Is each icon function clear with brief and descriptive images? (Criteria 7.4)

8. Aesthetic and minimalist design. Dialogues should not contain information which is irrelevant or rarely needed. Every extra unit of information in a dialogue competes with the relevant units of information and diminishes their relative visibility.

- Does the EduGate system provide small arrow icons to enable users to move between "next page" and "previous page"? (Criteria 8.1)

- Does the system consider the differences between novice and expert users, and support them appropriately through provision of multiple levels of error message detail? (Criteria 8.2)

- Does the system allow users to change language option from the main page without needing to move to the settings page? (Criteria 8.3)

- Does the system offer users the option of either clicking on boxes or using a keyboard shortcut? (Criteria 8.4)

9. Help users recognize, diagnose, and recover from errors. Error messages should be expressed in plain language (no codes), precisely indicate the problem, and constructively suggest a solution. 
- Does the system clearly differentiate text, lines images and background through clear use of colour and brightness? (Criteria 9.1)

- Are the icons and boxes clearly separated by white space? (Criteria 9.2)

- Is there a proportion between icons and background, meaning that icons stand out more clearly? (Criteria 9.3)

- Is it possible to recognize the icon's function from icons title, image or shape? (Criteria 9.4)

10. Help and documentation. Even though it is better if the system can be used without documentation, it may be necessary to provide help and documentation. Any such information should be easy to search, focused on the user's task, list concrete steps to be carried out, and not be too large.

- Is the EduGate provide sufficient assistance to the students? (Criteria 10.1)

- Is the helping messages appear to the student on appropriate time? (Criteria 10.2)

- Is the helping messages words clear and understandable by the students? (Criteria 10.3)

- Can the helping messages be offered when the students need them? (Criteria 10.4)

\subsection{Conducting Evaluation}

To evaluate the usability of the EduGate, the criteria designed in the previous section have been aggregated in a single form to represent a checklist that needs to be checked by the usability experts. The EduGate application was assessed by three experts in order to detect any potential usability problems. In selecting experts, it is ensured that they should satisfy a set of requirements. For example, some evaluators have $\mathrm{PhD}$ degree and they previously either studied HCI course or taught it. Fortunately, all selected evaluators have good experience and so are familiar with the use of the online academic portal applications.

\subsection{Analysis and Result}

To determine if a given academic portal application conforms to a given criterion, a voting system is used. Since the EduGate application was assessed by three experts it is easy to determine if the target criteria represent a potential usability problem or not. If at least two evaluators chose the No option, the EduGate application is classified to have usability problem with respect to the criteria under assessment. The detailed result is reported in the following section.

\section{Usability Problems of EduGate}

In order to evaluate the usability of the EduGate, the criteria designed in Section 4 have been aggregated in a single form to represent a checklist that needs to be checked by the usability experts. The EduGate was assessed by three experts in order to detect any potential usability problems. Fortunately, all selected evaluators have good experience and so are familiar with the use of the EduGate. To determine if the EduGate conforms to a given criterion, a voting technique is used [41]. Since the usability of EduGate was assessed by three experts it is easy to determine if the target criteria represent a potential usability problem or not. If at least two evaluators chose the "No" option, the EduGate is classified to have usability problem with respect to the criteria under assessment. Table 2 summarized the discovered usability problems of the EduGate. To improve the usability of the EduGate there follows a set of recommendations. 
The recommendations are motivated from analyzing the results of usability heuristic obtained from the three experts. To address the usability problems, 10 recommendations follow:

- Recommendation 1. To speed up the interaction between the student and EduGate application, it is recommended that the EduGate system to cater to both novice and advanced users by using keyboard function keys to perform some common actions. For example, to use F1 for course registration and F5 for view academic calendar.

- Recommendation 2. To give the students more flexibility, it is recommended to give the students the possibility of cancelling a given action during its running. For example, cancel course registration before the completion of all registration steps.

- Recommendation 3. It is quite useful if the EduGate system can alert students if they about to make an error. For example, it is suggested that the EduGate system shows a confirmation message to the student when pressing drop course.

- Recommendation 4. To provide sufficient assistance to the students, it is recommended that the EduGate offer some suggestions to help students solving the occurred problems and errors.

- Recommendation 5. For flexibility and efficiency of use the EduGate, it is recommended that the importance icons and menus are ordered based on the frequently used to allow students choosing them faster. For example, putting course drop after registration icons and courses' result at the end of the menu because it is used only once (i.e. at the end of semester).

- Recommendation 6. To offer more flexibility and freedom to students, it should consider the difference between novice and expert users. To do so, it is useful to use keyboard shortcut to perform some common actions. For example, to register a new course, it is recommended to use only $(\mathrm{N})$ instead of $\mathrm{Ctrl}+\mathrm{N}$ as a shortcut.

- Recommendation 7. To help the students recognize and diagnose, it is suggested that small icons and boxes be separated by clear white space.

- Recommendation 8. To provide sufficient assistance to the students, it is recommended that the EduGate give more detailed feedback. For example, instead give the students "the course has been successfully registered", "Introduction to programming course has been successfully registered".

- Recommendation 9. To provide clear and understandable helping messages, it is recommended that a simple words and detailed description be given to the student when for example the cursor hovers over icons or folders.

- Recommendation 10. It is recommended that the helping messages can be offered every time the students need them.

Table 2: Usability problems of EduGate

\begin{tabular}{|c|c|c|}
\hline Found Problem & Violated Heuristic & Suggested Improvement \\
\hline Criteria 1.4 & Visibility of system status & Recommendation1 \\
\hline Criteria 3.1 & User control and freedom & Recommendation 2 \\
\hline Criteria 5.2 & Error prevention & Recommendation 3 \\
\hline Criteria 5.4 & Error prevention & Recommendation 4 \\
\hline Criteria 7.2 & Flexibility and efficiency of use & Recommendation 5 \\
\hline Criteria 8.4 & Aesthetic and minimalist design & Recommendation 6 \\
\hline Criteria 9.2 & Help users recognize, diagnose, and recover from errors & Recommendation 7 \\
\hline Criteria 10.1 & Help and documentation & Recommendation 8 \\
\hline Criteria 10.3 & Help and documentation & Recommendation 9 \\
\hline Criteria 10.4 & Help and documentation & Recommendation 10 \\
\hline
\end{tabular}




\section{Evaluation}

This section seeks to evaluate the validity of the recommendations proposed in Section 5.0. It begins by briefly describing the method by which data was collected from real EduGate users (i.e. the students at King Saud University). After this, the process of data collection will be described in detail, from preparing the questionnaire to obtaining the result from the users. After this, the results are presented.

\subsection{Data Collection}

A questionnaire was designed to test how effectively the recommendations might improve the usability of EduGate. The questionnaire consisted of ten questions, one question for each recommendation.

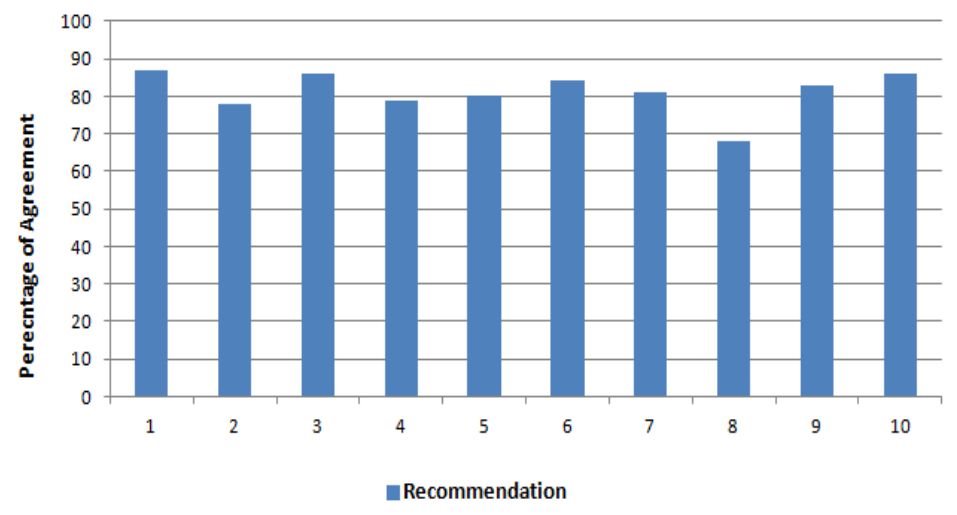

Figurer 3: Validity of suggested recommendations

Because of this, closed questions were chosen in this questionnaire, because they are quick and easy to answer, which is more likely to encourage participation in a questionnaire [42]. These types of questions also ensured that each of the respondents (i.e. students) would concentrate on the key issues of interest to this study. After preparing the questionnaire, it was published on Qualtrics.com, a free online survey software, making it accessible by EduGate users. Each participant was asked to answer ten questions, each of five answers on the Likert scale. The questionnaire was delivered to evaluate the recommendations made by this study in terms of their ability to improve the usability of the EduGate. The questionnaire was answered by 328 users and the results are produced and shown in Figure 3.

\subsection{Results and Findings}

The result reported in Figure 3 provides strong support for the popularity of our recommendations to improve the usability of the EduGate. On average, $81 \%$ of the participants have been agreed with the ten recommendations. That means the majority of students considered the proposed recommendations to be very important to make the EduGate more efficient and easier to use. While the participants have been agreed with providing the EduGate's users clear, understandable and on time helping messages, they prefer the messages to be short. Only $68 \%$ of the participants have agreed with the important of giving the users more detailed feedback, perhaps because the long feedback may make the message ambiguous and not understandable. In general, the results provide clear evidence about the validity of the proposed recommendations to enhance the usability of the EduGate. 


\section{CONCLUSiOnS}

In this paper, we have investigated and examined the usability of the EduGate. The heuristic evaluation method was used to inspect the usability problems of the EduGate. Ten usability problems were detected after the evaluation by three experts using the 10 principles proposed by Nielsen. Recommendations have been suggested to improve the usability of the EduGate and these recommendations have been evaluated by using the method of questionnaire to gather the views of students about study findings. One possible future area of investigation is to apply other usability methods to evaluate the usability of the EduGate. For example, testing and inquiry methods may be used, such as the asking-question protocol method [30], in which some participants could be asked to perform specific tasks and then ask them some questions relating to their interaction with the EduGate to allow the usability evaluator to understand their experience and their mental model of the system.

\section{REFERENCES}

[1] Tatnall A., Gateways To Portals Research. International Journal Of Web Portals, 1(1):1-15, 2009.

[2] Manouselis N., Kastrantas K., Alonso S.S, Caceres J., Ebner H., Palmér M., Architecture Of The Organic Edunet Web Portal. Ijwp. 1(1):71-91, 2009.

[3] Nielsen J., Molich R., Heuristic Evaluation Of User Interfaces. In: Proceedings Of The Sigchi Conference On Human Factors In Computing Systems. New York, Ny, Usa: Acm, P. 249-256, 1990.

[4] Preece J., Benyon D., University O., A Guide To Usability: Human Factors In Computing. 1st Ed. Boston, Ma, Usa: Addison-Wesley Longman Publishing Co., Inc.; 1993.

[5] Hix D., Hartson H.R., Developing User Interfaces: Ensuring Usability Through Product \& Process. New York, Ny, Usa: John Wiley \& Sons, 1993.

[6] Carroll J.M., Hci Models, Theories, And Frameworks: Toward A Multidisciplinary Science. Morgan Kaufmann, San Francisco, Usa, 2003.

[7] Preece J., Rogers Y., Sharp H., Benyon D., Holland S., Carey T., Human-Computer Interaction. 1st Edition. Wokingham, England ; Reading, Mass: Addison Wesley; 1994.

[8] Danino N., Heuristic Evaluation - A Step By Step Guide Article, Sitepoint. 2001, [Internet]: [Cited 2016 Aug 19]; Available From: Http://Www.Sitepoint.Com/Heuristic-Evaluation-Guide/

[9] Carroll J.M., Hci Models, Theories, And Frameworks: Toward A Multidisciplinary Science. Morgan Kaufmann, San Francisco, Usa, 2003.

[10] Rogers Y., Sharp H., Preece J., Interaction Design: Beyond Human - Computer Interaction. John Wiley \& Sons, New York, Ny, Usa, 2011.

[11] Shackel B., Richardson S.J., Human Factors For Informatics Usability. Cambridge University Press, Cambridge, Uk, 1991.

[12] Nielsen J., Usability Engineering. Morgan Kaufmann, San Francisco, Usa, 1994.

[13] Wd Iso. 9241-11. Ergonomic Requirements For Office Work With Visual Display Terminals (Vdts). 1998.

[14] Baecker R.M., Readings In Human-Computer Interaction: Toward The Year 2000. Morgan Kaufmann Publishers, San Francisco, Usa, 1995.

[15] Nielsen J., Usability 101: Introduction To Usability, 2003. Available From: Http://Www.Nngroup.Com/Articles/Usability-101-Introduction-To-Usability/

[16] Badre A., Shaping Web Usability: Interaction Design In Context. Addison-Wesley Professional, Boston, Ma, Usa, 2002.

[17] Friedman V., 10 Principles Of Effective Web Design, Smashing Magazine, [Internet], [Cited 2016 Aug 19];Available From: Http://Www.Smashingmagazine.Com/2008/01/31/10-Principles-OfEffective-Web-Design/

[18] Wallace M.D., Anderson T.J., Approaches To Interface Design. Interacting With Computers, 5(3):259-278, 1993.

[19] Pinelle D., Wong N., Stach T., Heuristic Evaluation For Games: Usability Principles For Video Game Design, In: Proceedings Of The Sigchi Conference On Human Factors In Computing Systems. Acm ,New York, Ny, Usa, P. 1453-1462, 2008.

[20] Dringus L.P, Cohen M.S., An Adaptable Usability Heuristic Checklist For Online Courses. In: Frontiers In Education, Proceedings 35th Annual Conference. P. T2h-6. 2005. 
[21] Wentz B., Lazar J., Usability Evaluation Of Email Applications By Blind Users. Journal Of Usability Studies. 6(2):75-89, 2011.

[22] Hussain, A. B., Abbas, S. A. A., Abdulwaheed, M. S., Mohammed, R. G., \& Abdullah Abdulhussein, A. Usability Evaluation Of Mobile Game Applications: A Systematic Review. International Journal Of Computer And Information Technology. 4(3): 547-551, 2015.

[23] Yousef, A. M. F., Chatti, M. A., Schroeder, U., \& Wosnitza, M. A Usability Evaluation Of A Blended Mooc Environment: An Experimental Case Study. The International Review Of Research In Open And Distributed Learning,16(2): 69-93, 2015.

[24] Iqbal, M., \& Warraich, N. F. Usability Evaluation Of An Academic Library Website: A Case Of The University Of The Punjab. Pakistan Journal Of Information Management \& Libraries (Pjim\&L), 2016.

[25] Arning, K., Himmel, S., \& Ziefle, M. "Overloaded, Slow, And Illogical” A Usability Evaluation Of Software For Product Manufacturing Processes With A Special Focus On Age And Expertise Of Cam Users. In Proc. International Conference On Competitive Manufacturing, Pp. 553-558, 2016.

[26] Choi, Y. M., \& Li, J. Usability Evaluation Of A New Text Input Method For Smart Tvs. Journal Of Usability Studies, 11(3), 2016.

[27] Thimbleby H., Interaction Walkthrough: Evaluation Of Safety Critical Interactive Systems. In: Interactive Systems. Design, Specification, And Verification. Springer; 2007.

[28] Maguire M., Bevan N., User Requirements Analysis. In: Hammond J, Gross T, Wesson J, Editors. Usability. Springer, Usa, 2002.

[29] Dumas J.S, Redish J., A Practical Guide To Usability Testing. Intellect Books, Exeter, Uk, 1999.

[30] Osterbauer C., Lackner M., Weinberger G., Usability In Chemical Engineering. Trends In Chemical Engineering. 13:1-15, 2010.

[31] Kellett M., How To Develop Children As Researchers: A Step By Step Guide To Teaching The Research Process. Sage, London, Uk, 2005.

[32] Nielsen J. Usability Inspection Methods. In: Conference Companion On Human Factors In Computing Systems. Acm , New York, Ny, Usa, P. 413-414. 1994.

[33] Nielsen J., 10 Heuristics For User Interface Design [Internet]. 2005 [Cited 2016 Aug 19];Available From: Http://Www.Nngroup.Com/Articles/Ten-Usability-Heuristics/

[34] Reeves T.C., Apedoe X., Hee Woo Y., Evaluating Digital Libraries: A User-Friendly Guide [Internet]. 2005 [Cited 2016 Aug 19];Available From: Http:/Www.Dpc.Ucar.Edu/Projects/Evalbook/

[35] Polson P.G., Lewis C., Rieman J., Wharton C., Cognitive Walkthroughs: A Method For TheoryBased Evaluation Of User Interfaces. Int. J. Man-Mach. Stud. May 36(5):741-773. 1992.

[36] Rieman J., Franzke M., Redmiles D., Usability Evaluation With The Cognitive Walkthrough. In: Conference Companion On Human Factors In Computing Systems. Acm, New York, Ny, Usa; P. 387-388, 1995.

[37] Bias R.G., The Pluralistic Usability Walkthrough: Coordinated Empathies. In Usability Inspection Methods. In: Nielsen J, Mack R.L, Editors. New York, Ny, Usa: John Wiley \& Sons, Inc.; P. 63-76, 1994.

[38] Collura M., Human-Computer Interaction And Main Principles To Design Practice Human-Centred System. Management And Industrial Engineering Department. Universita Della Calabria, Cosenza. 2006.

[39] Powell R.A, Single H.M., Focus Groups. Int J Qual Health Care. Jan 1;8(5):499_ 504. 1996.

[40] Kantner L., Sova D.H, Rosenbaum S. Alternative Methods For Field Usability Research, In Proceedings Of The 21st Annual International Conference On Documentation. New York, Ny, Usa: Acm; 2003.

[41] Tan C.K., Amin S.H., Mamat R., Using Voting Technique In Mobile Robot Behavior Coordination For Goal-Directed Navigation. Jurnal Teknologi, 36(1):55-70, 2012.

[42] Sonnekus I.P., Pieterse V., Ascertaining Personal Qualities Of Computer Educators Who Are Destined To Achieve Results (Cedars). International Journal Of Learning. The Learner Collection, Vol. 10, 2010. 